\title{
The Auroral Spectrum and the Upper Atmosphere.
}

\section{By Prof. L. VEgard, University of Christiania.}

THE aurora borealis is produced by the action of electric radiation from outer space upon the upper atmosphere. Height measurements have shown that such radiation usually penetrates to a height of I00-x०5 $\mathrm{km}$. Most auroral forms have their maximum light intensity at a height of $10-20 \mathrm{~km}$. from the bottom edge, and by far the greater part of the light emitted from the aurora comes from a height interval of roo$130 \mathrm{~km}$. The upper limit varies very much. Usually we can only follow an aurora up to an altitude of $I_{30-x} 5^{\circ}$ $\mathrm{km}$., but the ray-forms may be seen much higher. The greatest altitude at which isolated auroral "rays" may appear seems to increase towards lower latitudes. Near the auroral zone no rays are observed higher than 300-350 km. At Christiania, however, Störmer finds that isolated rays may reach altitudes of $75^{\circ} \mathrm{km}$.

Practically all auroral light is emitted from the matter present in the atmosphere, and not from the carriers of the cosmic rays; for none of the principal lines in the auroral spectrum shows any Doppler effect. The character of the light must be a function of the properties of the electric radiation and the constitution of the upper strata of the atmosphere.

The auroral spectrum has attracted much attention from many observers; but when, in 1910, in connexion with investigations of the properties of the cosmic electric rays, I sought for information on this point from the auroral spectrum, I found that practically all measurements were too inaccurate for identification of the lines, and there were as many-or even more-interpretations of the spectrum as there were observers. The analysis and interpretation of the auroral spectrum at that time was, therefore, an open problem.

In I9I2-I3 I commenced observations at Bossekop in Finmarken on the auroral spectrum. On this first expedition I used a spectrograph which combined a fairly high light power with a considerable dispersion, and I had also a spectroscope at my disposal. With the spectroscope I observed the prominent green line, and the best series of observations gave $\lambda=5577$. With the spectrograph I obtained the green line and six lines in the blue and violet; and the latter were found to be identical with prominent lines in the negative band spectrum of nitrogen. This result has recently been confirmed by Lord Rayleigh. As the different determinations of the green line showed some variations, I undertook new measurements at Christiania with a spectroscope of greater dispersion. The first series gave $\lambda=5578.4 \AA$, and the second and most extensive series $\lambda=5577.6 \AA$. These values are in good agreement with those obtained from the night sky by Slipher, and recently by Babcock, at the Mount Wilson Observatory.

Although, however, the wave-length of the green line was found with great accuracy, its origin seemed as mysterious as ever. In order to get on the track of the origin of the green line, I determined to commence more systematic investigations on the auroral spectrum. Suitable spectrographs were constructed and mounted on the roof of the Geophysical Institute at Tromsö. Observations were carried on during the winter $1922-23$, and also during last winter.
The first winter's work gave a number of good spectrograms, and 35 lines and bands were measured in the visible and ultraviolet parts of the spectrum. With the exception of the green line and three faint lines or bands, the lines were identified with known nitrogen lines. The auroral spectrum is not merely remarkable on account of the green line, but the other part is also of a most singular type. Out of the enormous number of lines and bands in the nitrogen spectrum, only some very few lines or band-heads are separated out in the auroral spectrum.

Although the stronger lines in the auroral spectrum were greatly over-exposed, no trace of hydrogen or helium was found. The view previously current that the atmosphere above roo km. should mainly consist of light gases had, therefore, to be abandoned. It might be suggested that hydrogen, helium, or the hypothetical "geocoronium" produced the green line; but it is then curious that hydrogen and helium did not show any of their ordinary lines. By comparing the intensity of the green line with that of the known nitrogen lines of the auroral spectrum on spectrograms corresponding to the lower and upper limit of radiant aurora, it was shown that the green line could not originate from any of these light gases; for instead of increasing upwards, the intensity of the green line was found to be somewhat greater at the lower limit.

This fact, in connexion with the other fact that nearly the whole spectrum consisted of nitrogen lines, gave a strong argument in favour of the view, which I expressed so long ago as I9IO, that the green line also belongs to nitrogen, and is emitted under the special physical conditions existing in the auroral region.

Independent of the view which might be taken with regard to the origin of the green line, we had to assume nitrogen to be a prominent component of the atmosphere to its very upper limit. To explain this we could assume either an increasing temperature upwards, or that the nitrogen must be electrically charged and be driven upwards by electric forces. The character of the spectrum and other reasons made me give up the first assumption, and following up the second I found that an electrified atmosphere in a highly ionised state could not exist in the form of ordinary gas, and so I was led to assume that the nitrogen at a very low temperature was condensed into clusters or small crystals.

This hypothesis, which at first might seem somewhat startling and contrary to previous conceptions, proved to be a most fertile one. First of all it gave for the auroral region a physical state which would explain why the auroral spectrum had not yet been reproduced in laboratory experiments; it gave possibilities for explaining the conspicuous colour changes of the aurora; and also simple explanations of other cosmic phenomena such as the twinkling of stars, the blue sky, and the zodiacal light.

As a consequence of this hypothesis it would follow that the auroral spectrum should be produced when this atmosphere of crystalline nitrogen dust was bombarded with electric rays. These conditions, which are very difficult to reproduce exactly, might be at any

NO. 2846 , voL. I I 3 ] 
rate approximately reproduced by bombarding solid nitrogen with such electric rays as can be produced in the laboratory. Through the generosity of Prof. Kamerlingh Onnes I was able to take up experiments of this kind at the Cryogenic Laboratory of Leyden. I have thus been able to make a first series of

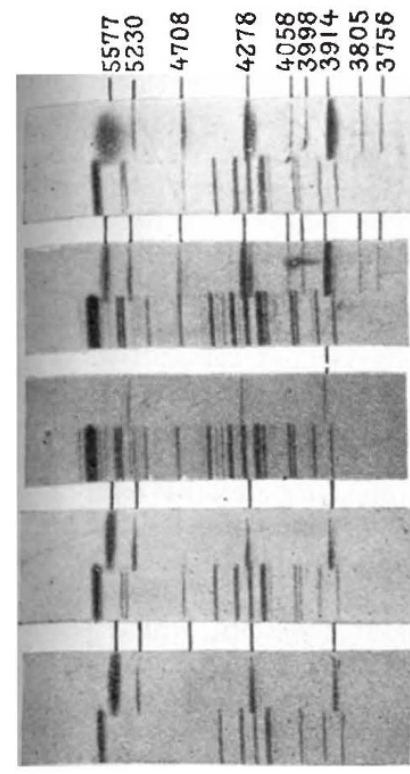

FIG. $x$. experiments on the light produced when solid nitrogen is bombarded with cathode rays, and these experiments gave, indeed, the very light effect which I expected. . $^{1}$

Rays from a Wehnelt cathode were made to fall on a layer of solid nitrogen formed on a copper surface cooled with liquid hydrogen. I took five spectra (Fig. I) corresponding to various velocities of the rays. At a tension of 75 volts, only the strongest heads of the negative band spectrum were observed. At 200 volts (Fig. I, No. I) there appeared also a green line near the blue part $\left(\mathrm{N}_{2}\right)$ and a diffuse band near the yellow part.

When the potential was raised to 500 volts, the layer of nitrogen became brilliantly luminous with green colour, and in addition to $\mathrm{N}_{2}$, a broad line or narrow band $\left(\mathrm{N}_{1}\right)$ now appeared with great intensity (Fig. I, No. 2). By lowering the potential to about $35^{\circ}$ volts, $\mathrm{N}_{1}$ disappeared (Fig. $\mathrm{x}$, No. 3), but by increasing it to 700 volts (Fig. I, No. 4) and 750 volts (No. 5) the light intensity, and especially that of $\mathrm{N}_{1}$, increased enormously. The line $\mathrm{N}_{1}$ now dominates the spectrum, just as the green line (5577) is predominant in the auroral spectrum.

To get ån impression of the relative light intensity at the different potentials it may be mentioned that in Fig. I the times of exposure of the spectra are 30,15 , Io, 5 and 5 minutes respectively.

When the cathode ray bombardment was stopped, the solid nitrogen layer remained luminous for more than five minutes, and it is in this connexion of interest to remember that an afterglow of about the same duration is also observed for the aurora. Measurements showed that the green auroral line (5577) is situated in the broad line $\mathrm{N}_{1}$. The line $\mathrm{N}_{2}$, which was found to have a wavelength about $5230 \AA$, coincides with a second fairly conspicuous green line in the auroral spectrum.

In Fig. 2 three auroral spectra (Nos. I, 2, 3) and one spectrum from solid nitrogen (No. 4) are reproduced. No. $I$ is taken with a large quartz spectrograph. The

1 A more complete account will be found in the Proc. of the Roy. Acad. of Sciences, Amsterdam.

$$
\text { NO. } 2846 \text {, vOL. I I } 3]
$$

others are enlarged copies from spectrograms taken with the same small glass spectrograph. Nos. $x$ and 2 are taken on Imperial Eclipse plates, No. 3 on panchromatic and No. 4 on orthochromatic plates.

The agreement between the auroral spectrum and that of solid nitrogen is not restricted to the green part; but we see that also in the blue and violet the crystalline nitrogen gives just the reduction in the number of lines which is so characteristic of the auroral spectrum.

The comparison shows that the typical auroral spectrum is emitted from solid nitrogen, and thus my hypothesis with regard to the constitution of the upper atmosphere has been confirmed.

The high velocity necessary for producing the lines $\mathrm{N}_{1}$ and $\mathrm{N}_{2}$, and the very great variability of their intensity, are most remarkable facts from a physical point of view, and this variability may help us to understand many of the colour changes of the aurora. The afterglow indicates that the new light effect is a kind of phosphorescence ; but to make clear its physical nature more experimental material is needed.

The new effect opens out a new field of research equally interesting from a cosmical and from a purely physical point of view. A more detailed study of the light emitted from solid nitrogen at various temperatures down to that of liquid helium may enable us to determine more exactly the temperature interval of the auroral region, and it is possible that, at an extremely low temperature, the broad line $N_{1}$ will become sharp. Experiments with rays of different velocities and carriers may give us information about the velocities and physical nature of the cosmic rays producing the auroræ.

If also other gases, e.g. hydrogen, oxygen, argon, neon, ammonia, nitric oxide, carbon monoxide, cyanogen, etc., in the solidified state under the action of electric rays give similar light effects to those of nitrogen, we may hope in this way to explain the

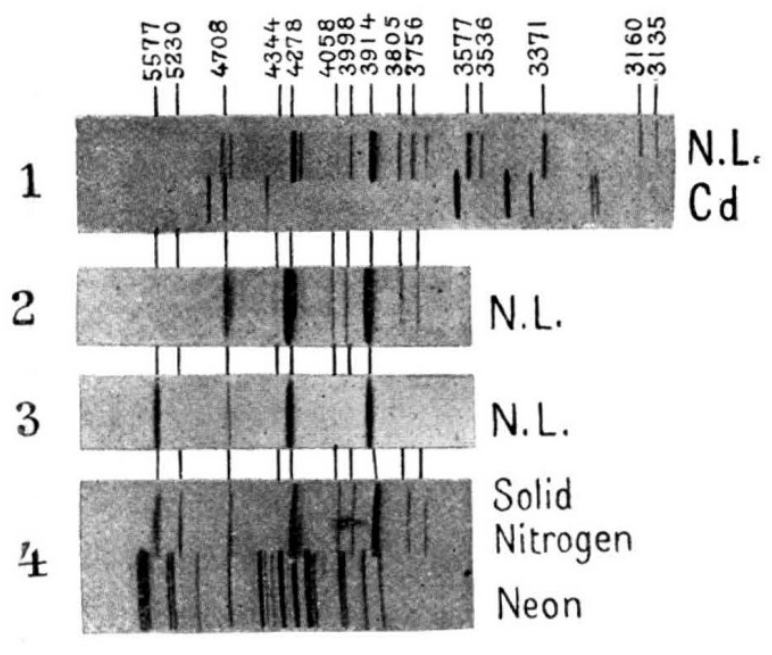

FIG. 2.

nebular lines and acquire a definite knowledge with regard to the constitution of nebulæ. Perhaps "nebulium" will then pass the same way as "geocoronium."

I am continuing experiments in the directions here indicated at the Kamerlingh Onnes Laboratory at Leyden. 